\title{
Characterization of cognitive deficits in spontaneously hypertensive rats, accompanied by brain insulin receptor dysfunction
}

\author{
Edna Grünblatt ${ }^{1,2,3,5^{*}}$, Jasmin Bartl ${ }^{1}$, Diana-Iulia Iuhos $^{3}$, Ana Knezovic ${ }^{4}$, Vladimir Trkulja ${ }^{4}$, Peter Riederer ${ }^{3}$,
} Susanne Walitza ${ }^{1,2}$ and Melita Salkovic-Petrisic ${ }^{4}$

\begin{abstract}
Background: The spontaneously hypertensive rat (SHR) has been used to model changes in the central nervous system associated with cognitive-related disorders. Recent human and animal studies indicate a possible relationship between cognitive deficits, insulin resistance and hypertension. We aimed to investigate whether cognitively impaired SHRs develop central and/or peripheral insulin resistance and how their cognitive performance is influenced by the animal's sex and age as well as strains used for comparison (Wistar and Wistar-Kyoto/WKY).

Methods: Three and seven-month-old SHR, Wistar, and WKY rats were studied for their cognitive performance using Morris Water Maze (MWM) and Passive Avoidance tests (PAT). Plasma glucose and insulin were obtained after oral glucose tolerance tests. Cerebral cortex, hippocampus, and striatum status of insulin-receptor (IR) $\beta$-subunit and glycogen synthase kinase-3 $\beta$ (GSK3 $\beta$ ) and their phosphorylated forms were obtained via ELISA.

Results: SHRs performed poorly in MWM and PAT in comparison to both control strains but more pronouncedly compared to WKY. Females performed poorer than males and 7-month-old SHRs had poorer MWM performance than 3-month-old ones. Although plasma glucose levels remained unchanged, plasma insulin levels were significantly increased in the glucose tolerance test in 7-month-old SHRs. SHRs demonstrated reduced expression and increased activity of IRß-subunit in cerebral cortex, hippocampus, and striatum with different regional changes in phospho/total GSK3 $\beta$ ratio, as compared to WKYs.

Conclusion: Results indicate that cognitive deficits in SHRs are accompanied by both central and peripheral insulin dysfunction, thus allowing for the speculation that SHRs might additionally be considered as a model of insulin resistance-induced type of dementia.
\end{abstract}

Keywords: Age, Control strain, Gender, Glycogen synthase-kinase $3 \beta$, Insulin resistance, Learning and memory, Spontaneously hypertensive rat

\section{Background}

The spontaneously hypertensive rat (SHR) was inbred from the Wistar-Kyoto rat strain (WKY) [1]. SHRs were initially developed as a model for hypertension but were eventually shown to develop pathologies at the metabolic, behavioral, and cognitive level. The metabolic pathology is manifested as systemic insulin resistance

\footnotetext{
*Correspondence: edna.gruenblatt@kjpdzh.ch

'University Clinics of Child and Adolescent Psychiatry, University of Zurich,

Neumuensterallee 9, 8032 Zurich, Switzerland

${ }^{2}$ Neuroscience Center Zurich, University of Zurich and ETH Zurich, Zurich, Switzerland

Full list of author information is available at the end of the article
}

[2]; the behavioral symptoms resemble attention-deficit hyperactivity disorder (ADHD) (hyperactivity, impulsivity, impaired ability to withhold responses, and poorly sustained attention) [3-5]; and the cognitive pathology is manifested as learning and memory deficits [6-8]. Several studies have explored the link between an insulin resistant brain state and cognitive impairment, particularly in dementia of the Alzheimer type as reviewed elsewhere [9-11]. Insulin and insulin receptors (IR) in the brain have been shown to regulate glucose metabolism [12], as expected, but IR also triggers complex signaling pathways in the brain [13-17]. These pathways involve 
glycogen synthase-kinase-3 (GSK3) inhibition, via protein kinase $B(A k t / P K B)[16,17]$. In addition, IR influences the accumulation/degradation of amyloid- $\beta$ and tau protein, the major neuropathological hallmarks of the memory loss developed in Alzheimer's disease (AD) [13-15]. We aimed to investigate whether cognitively impaired SHRs develop a central insulin resistance in parallel to the peripheral one, but data published so far on cognitive performance in the SHR model have indicated that measurements were done using different cognitive tests, comparing different control strains, using animals of different ages and not always done using both sexes [3, 18-32]. Since these factors might interfere with cognitive performance in rats, we have additionally explored their possible influence on learning and memory functions in the SHR model.

In brief, following the comparison between different control strains and sexes which showed that female SHRs demonstrate more pronounced cognitive deficits than males, particularly in comparison to the WKY controls, our studies showed that cognitive deficits in female SHRs are accompanied by peripheral and central insulin resistance in 7- but not in 3-month old animals in comparison to WKY controls.

\section{Methods}

\section{Animals}

The experiments were performed on Wistar SHR and 2 normotensive control strains, Wistar-Kyoto (WKY) and Wistar rats, all purchased from Charles River Laboratories (Kisslegg, Germany). Animals were acclimated for 2 weeks before cognitive testing which was performed at the Department of Pharmacology, University of Zagreb School of Medicine (Zagreb, Croatia). Animals consumed standardized food pellets and water ad libitum.

\section{Ethics}

All procedures were performed under the guidance of the Principles of Laboratory Animal Care (NIH Publication No. 80-23, revised in 1996) and in accordance with the European Communities Council Directive of 24. November 1986 (86/609/EEC) and the Croatian Act on Animal Welfare (NN 135/2006). All experiments were approved by the University of Zagreb School of Medicine (Licence No.04-1343-2006).

\section{Experimental design}

Three different experiments were performed in vivo to explore the effect of (1) control rat strain, with 12-week male SHR, WKY and Wistar rats, (2) sex, with 12-weekold female and male SHR and WKY rats, and (3) age, with 12- and 28-week-old (i.e. 3- and 7-month-old) female SHR and WKY rats. Animal age in the third experiment corresponded to young adult and older, "middle-aged" humans [33] as well as represented the periods of rising blood pressure (3 months) and sustained/chronic hypertension (7 months) [34, 35]. There were 10 animals per group in all experiments.

\section{Cognitive testing}

Morris Water Maze (MWM) swimming test

The MWM tested learning ability and spatial memory as previously described and used in our experiments $[36,37]$ (details provided in the Additional file 1). The time needed to find the platform (seconds) and the number of errors (incorrect entries into quadrants with no platform) were recorded in training trials during 4 consecutive days while the time spent searching for the platform after entering quadrant IV and number of errors were recorded in the probe trial that followed.

\section{Passive Avoidance test (PAT)}

The step-through PAT was performed two days after finishing the MWM test and exploited a fear-motivated tendency of a rat to escape from an illuminated area into a dark area as previously described and used in our experiments [36, 37]. Latency time (seconds) before entering the dark area was recorded on the third testing day. A more detailed PAT method is provided in the Additional file 1.

\section{Oral glucose tolerance test}

The OGTT was performed after the rats were under deep chloral hydrate $(300 \mathrm{mg} / \mathrm{kg}$ i.p.) anesthesia, in accordance with the ethical requirements for laboratory animal procedures of the Medical School University of Zagreb. Blood samples were sequentially collected from the tail vein ( $50 \mu \mathrm{L}$; between 8 and 12 a.m.) for plasma glucose measurements at baseline, 30, and $60 \mathrm{~min}$ after the challenge. The post-OGTT cut-off time of $60 \mathrm{~min}$ corresponded to the duration of anesthesia from a single chloral hydrate dose. Animals were sacrificed after the last blood withdrawal still in deep anesthesia, 2 days after last cognitive testing.

\section{Preparation of brain regions}

Macrodissection of three brain regions (frontal cortex, striatum, and hippocampus) was performed on a cold plate $\left(4{ }^{\circ} \mathrm{C}\right)$ by a standard procedure and according to the rat brain atlas [38]. The brain samples were snap frozen and stored at $-80^{\circ} \mathrm{C}$ until further analysis.

\section{Brain homogenates}

Frozen brain tissue from each rat was weighed, and a 4-fold concentration of RIPA buffer (Sigma-Aldrich, Schelldorf, Germany) was added with protease and phosphatase inhibitors (Sigma-Aldrich, Schelldorf, Germany). Samples were homogenized via ultrasonic disruption (15\% amplitude, $10 \mathrm{~s}$ ) at $4{ }^{\circ} \mathrm{C}$. After homogenization, the 
probes were centrifuged at $44,000 \times g$ at $4{ }^{\circ} \mathrm{C}$ for $10 \mathrm{~min}$. Clear supernatant (which contained proteins) was collected into a fresh tube for further analysis.

\section{Protein concentration}

For each sample, protein concentrations were evaluated with the standard Bradford method for protein measurement [39].

\section{Biochemistry}

Plasma glucose concentrations were measured with a commercial kit (Glucose-PAP Test, Herbos Diagnostics) and the glucose oxidase method. Plasma insulin levels were assessed with a commercial kit for an Enzyme-Linked Immunosorbent Assay (ELISA; Crystal Chem Inc, IL, USA; Cat. No. 90060), which was more sensitive for quantitative analysis (sensitivity $<0.8$ units $/ \mathrm{ml}$ ) than a Western Blot assay. ELISAs were used to evaluate brain expression levels of the insulin receptor (IR) $\beta$-subunit (total), the two forms of the phosphorylated-IR $\beta$ subunit, $\mathrm{pTyr}^{1162 / 1163}$ and pTyr $^{1158}$ (Calbiochem, Darmstadt, Germany; CBA039, CBA038, and Invitrogen $\mathrm{GmbH}$, Darmstadt, Germany; KHR9121, respectively), the glycogen synthase-kinase (GSK)-3 $\beta$-subunit (total), and the phosphorylated form of GSK-3 $\beta$, pSer $^{9}$ (Calbiochem, Darmstadt, Germany; CBA068 and CBA069, respectively). All ELISAs were performed as recommended by the manufacturer. In the ELISA tests, brain homogenates were run in parallel with standards for concentration evaluations.

\section{Statistical analysis}

The statistical analyses of all data were performed with StatView for Windows (SAS Institute Inc., Cary, NC, USA; Version 9.3). Data from the MWM training trials were analyzed by fitting general(ized) linear mixed models (Poisson link for the number of errors, normal for escape latency. Instead of adjustment for multiple comparisons, which we considered too conservative, main effects (across all days or both genders/strains) of strain and gender are given with $95 \%$ confidence interval (CI); contrasts arising from interactions with up to 4 cross-products (strain*gender across all days, strain*age across all days) are given with $97.5 \% \mathrm{CI}$; contrasts arising from strain*(sex or age)*day interaction are given with $99 \% \mathrm{CI}$ (the significance level $p<0.05,<0.025$ or $<0.01$, respectively). Additionally, the multifactorial analysis of variance (MANOVA), followed by the analysis of Post-Hoc Scheffé test, and the Mann-Whitney $U$-test, was used. The significance level was set to $\mathrm{p}<0.05$.

\section{Results}

\section{Cognitive deficits}

After demonstrating that SHR rats performed poorly in MWM training trials regardless of the control strain
(WKY or Wistar) $(p<0.05)$, but that the difference was more pronounced compared to WKY $(p<0.05)$, WKY rats were used as a control in further experiments, which consistently showed that SHRs performed worse regardless of sex $(p<0.05)$ or age $(p<0.05)$, as presented in Additional file 2: Figure S2 \& Additional file 3: Figure S3.

As we could show that the most pronounce differences in cognitive deficits in training trials were in female SHR compared to female WKY $(p<0.05)$, and in some extent due to age $(p<0.05)$ (Additional file 3: Figure S3), the cognitive performance of 3 - and 7 month old female SHR and WKY rats was further recorded in the MWM probe trials. Both SHR age groups demonstrated significant deficits in spatial memory; they spent less time swimming in the quadrant from which the platform has been removed $(p<0.0001$; Fig. 1a) and made more errors than controls (7-month-old SHRs, $p<0.05$; 3-month-old SHRs, $p=0.06$; Fig. 1b). Female SHRs of both ages also showed marked deficits in fear-motivated memory in the PAT. They demonstrated much lower post-shock latency times than WKY controls $(p<0.0001$, Fig. 1c), with no age-related effect being detected.

\section{Insulin receptor signaling in the brain}

Based on the results of cognitive testing, female SHRs were further used to investigate whether cognitive deficits are accompanied by alterations of insulin signaling in the brain, when compared to WKY controls.

\section{Brain IR $\beta$ expression levels}

SHRs had, in general, lower total IR $\beta$ subunit levels than WKY rats in all brain regions, particularly in the hippocampus and striatum (Fig. 2a) but showed agedependent increment in IR $\beta$ level in the frontal cortex only ( $p<0.05$, Fig. $2 \mathrm{a})$ in contrast to region-dependent variability in the total IR $\beta$ levels with aging in WKY controls (frontal cortex-increased $/ p<0.05 /$, hippocampus-decreased $/ p<0.05 /$, striatum-unchanged, in 7- compared to 3-month-old animals, respectively) (Fig. 2a).

\section{Brain IR activity}

IR activity expressed as the ratio of phosphorylated IR $\left(\mathrm{pTyr}^{1162 / 1163}\right.$ and $\mathrm{pTyr}^{1158}$ ) to total IR $\beta$ (higher ratio indicating higher IR activity) was significantly increased in all investigated brain regions in 7-compared to the 3month-old animals in both SHRs and WKYs but the magnitude of age-dependent increment was larger in SHRs than in WKYs (Fig. 2b). Compared to WKYs, SHRs had significantly higher IR activity at both ages in the hippocampus and striatum and at 7-month of age in the frontal cortex $(p<0.05$; Fig. 2b). 


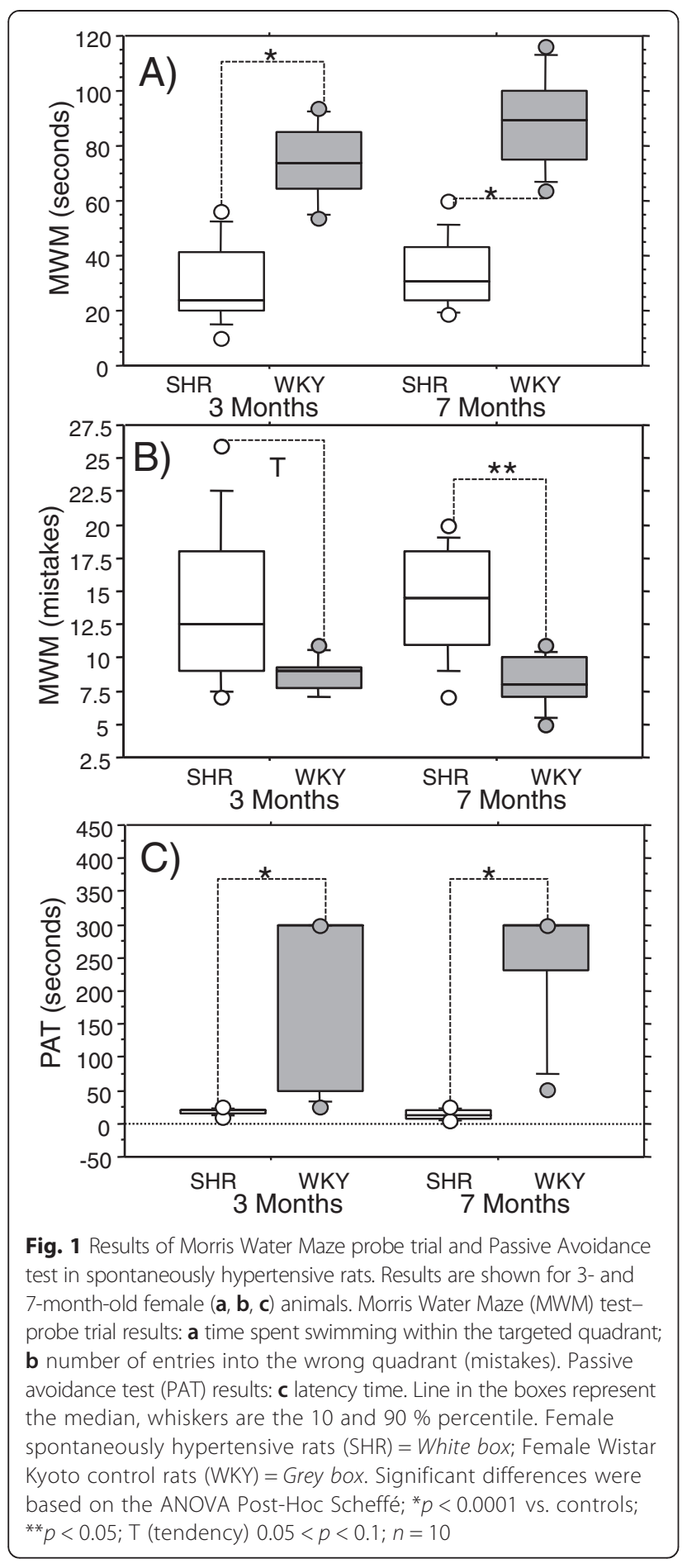

Brain GSK3 $\beta$ expression levels and activity

Total GSK3 $\beta$ level in the frontal cortex $(p<0.0005)$, hippocampus $(p<0.0001)$ and striatum $(p<0.005)$ was significantly lower in 3- compared to 7-month-old SHRs (Fig. 3a) while WKYs expressed such age-dependent difference only in the hippocampus $(p<0.05$; Fig. 3a). Since GSK3 $\beta$ activity is regulated mainly via phosphorylation of the serine-9 residue, we determined its relative
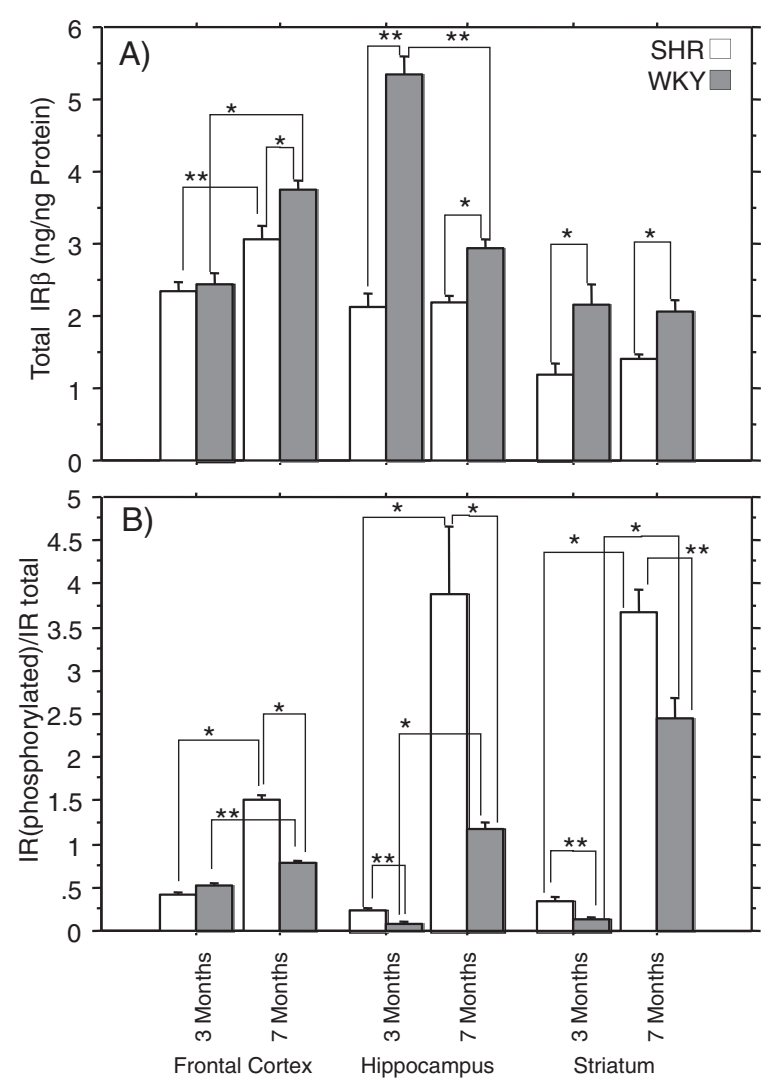

Fig. 2 Brain insulin receptor- $\beta$-subunit levels and activity in spontaneously hypertensive and Wistar Kyoto control rats. (a) Insulin receptor (IR) $\beta$-subunit levels and (b) the ratio of IR $\beta$ phosphorylated $\left(\mathrm{pTyr}^{1158}\right.$ and $\mathrm{pTyr}^{1162 / 1163}$ )/total IR $\beta$ are shown for different brain regions of 3- and 7-month-old spontaneously hypertensive rats (SHRs $=$ White $)$ and Wistar Kyoto control rats $($ WKY $=$ Grey). Columns represent the mean \pm SEM Significant differences were based on the ANOVA Post-Hoc Scheffé test; ${ }^{*} p<0.0001 ;{ }^{* *} p<0.05 ; n=10$

activity expressed as the ratio of the phosphorylated (inactive) to total GSK3 $\beta$ form (low ratio indirectly suggests high GSK3 $\beta$ activity). Age-dependent tendency of decrement in this ratio (increased GSK3 $\beta$ activity) was observed in both SHRs and WKYs with significance reached only in 7-month-old animals but with some regional specificity; SHRs - frontal cortex and striatum $(p<0.05)$, WKY - frontal cortex $(p<0.005)$ and hippocampus $(p<0.0001)$ (Fig. 3b).

\section{Plasma glucose and insulin levels}

Both 3- and 7-month-old SHRs showed a significant increase in plasma glucose after 30 and 60 min compared to the baseline levels in the oral glucose tolerance test (OGTT; Fig. 4a and b). A similar time-pattern effect was detected in WKYs with the exception of the $60 \mathrm{~min}$ time-point in 7-month-old WKYs when glucose levels were not significantly different from the respective baseline values (Fig. 4a and b). In contrast, the time-course 

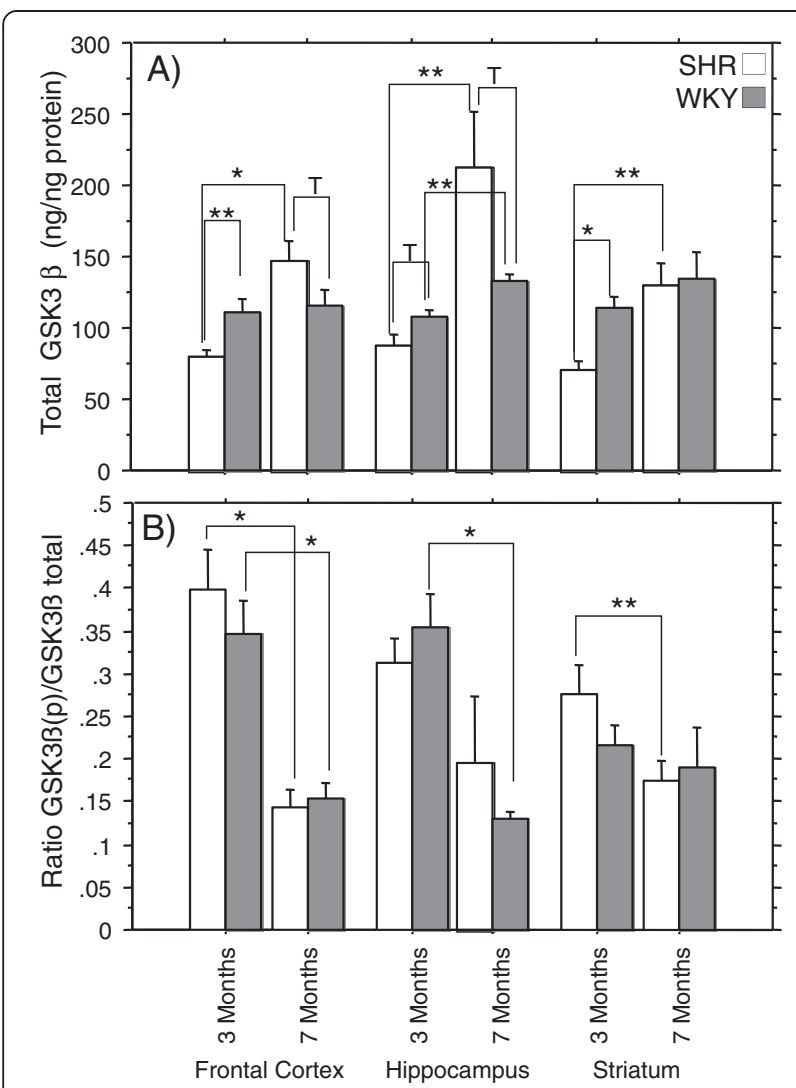

Fig. 3 Brain glycogen synthase-kinase-3 $\beta$-subunit levels and activity in spontaneously hypertensive and Wistar Kyoto control rats. (a) Glycogen synthase-kinase-3 $\beta$-subunit (GSK3 $\beta$ ) levels and (b) the ratio of GSK3 $\beta$ phosphorylated $\left(\mathrm{pSer}^{9}\right) / \mathrm{GSK} 3 \beta$ total are shown for different brain regions of 3- and 7-month-old spontaneously hypertensive rats (SHRs $=$ White) and Wistar Kyoto control rats $(\mathrm{WKY}=$ Grey). Columns represent the mean \pm SEM. Significant differences were based on the ANOVA Post-Hoc Scheffé test; * $p<0.005$; ${ }^{* *} p<0.05 ; 0.05<T<0.1 ; n=10$

of the plasma insulin levels showed opposite effects in the 3- and 7-month-old rats. In 3-month-old animals, SHRs showed only modest increase in plasma insulin with borderline significant differences at $60 \mathrm{~min}$ compared to the baseline levels, while insulin plasma levels in WKYs were significantly increased at both timepoints compared to their baseline level (Fig. 4c). In the 7-month-old animals, both the SHRs and WKYs showed significant increases in plasma insulin at the $60 \mathrm{~min}$ time-point only, compared to their baseline levels (Fig. 4d). Analysis of the area under the curve (AUC) values of plasma glucose and insulin (Fig. 4e-f) in SHRs and WKYs of both ages pointed to an age-dependent disruption of glucose metabolism for both groups. But to the contrary, plasma insulin AUC was significantly increased with age in the SHR animals, while no significant age-dependent difference was observed in WKY controls.

\section{Discussion}

Consistent with previous reports [7, 40-42], this study confirmed that the SHR model develops significant memory and learning deficits, which was a prerequisite for the study's main aim: to explore whether cognitive deficits in SHRs are accompanied by peripheral/central metabolic abnormalities. Considering literature data discussing that selection of corresponding control group (rat strain and usage of inbred, e.g. Wistar-Kyoto/WKY/, or outbred, e.g. Wistar, Sprague-Dawley/SD/animals) as well as age and sex might affect the results of cognitive testing $[23,25,43]$, we investigated the cognitive profiles of the optimal control strain and SHR gender and identified those which demonstrated most pronounced difference between the control and SHR group, to be used in exploring the metabolic changes in this model.

Our experiments (Additional file 2: Figure S2 \& Additional file 3: Figure S3) provided evidence that the SHR model performed poorly compared to control regardless sex, but females performed worse than males. Some literature data have suggested that hyperactivity behavior might influence the cognitive performance of SHRs and that locomotor activity (distraction) could be a confounding factor in spatial memory tasks [24], for example, in hyperactive female SHRs [32]. Although increased locomotor activity might act as a distraction in achieving a goal, hyperactivity in a cognitively normal rat might also lead to acheiving this goal in a more focused, direct way than in a rat with normal locomotor activity (which was not the case in our experiments). The present data (Additional file 2: Figure S2), however, strongly suggest that the observed deficit manifested as an increased number of errors was not due to hyperactivity: compared to controls, SHR rats made more errors (Additional file 2: Figure S2a, d) and had longer latency times (Additional file 2: Figure S2b, e), but time spent per one error was the same in all strains (Additional file 2: Figure S2c), and with adjustment for the number of errors, latency times did not differ between the SHR and control strains (Additional file 2: Figure S2F). Had hyperactivity (as a distraction factor) been the cause of the increased error rate, one would have expected to see reduced latency times per one error (e.g., faster swimming due to locomotor hyperactivity), or extended latency times per error (e.g., "non-focused" swimming due to hyperactivity), but not the same latency time per error. Since cognitive deficits in our experiments with cognitively more impaired female SHRs were more pronounced in comparison to WKY than to Wistar animals, female SHRs and WKYs were used for further exploration of metabolic abnormalities. WKY and Wistar strains have been frequently used as a control for memory testing in SHRs [22, 23, 25, 43, 44], but usually not in the same experiment with the exemption of the work of Gattu et al. [45, 46] which showed cognitive 


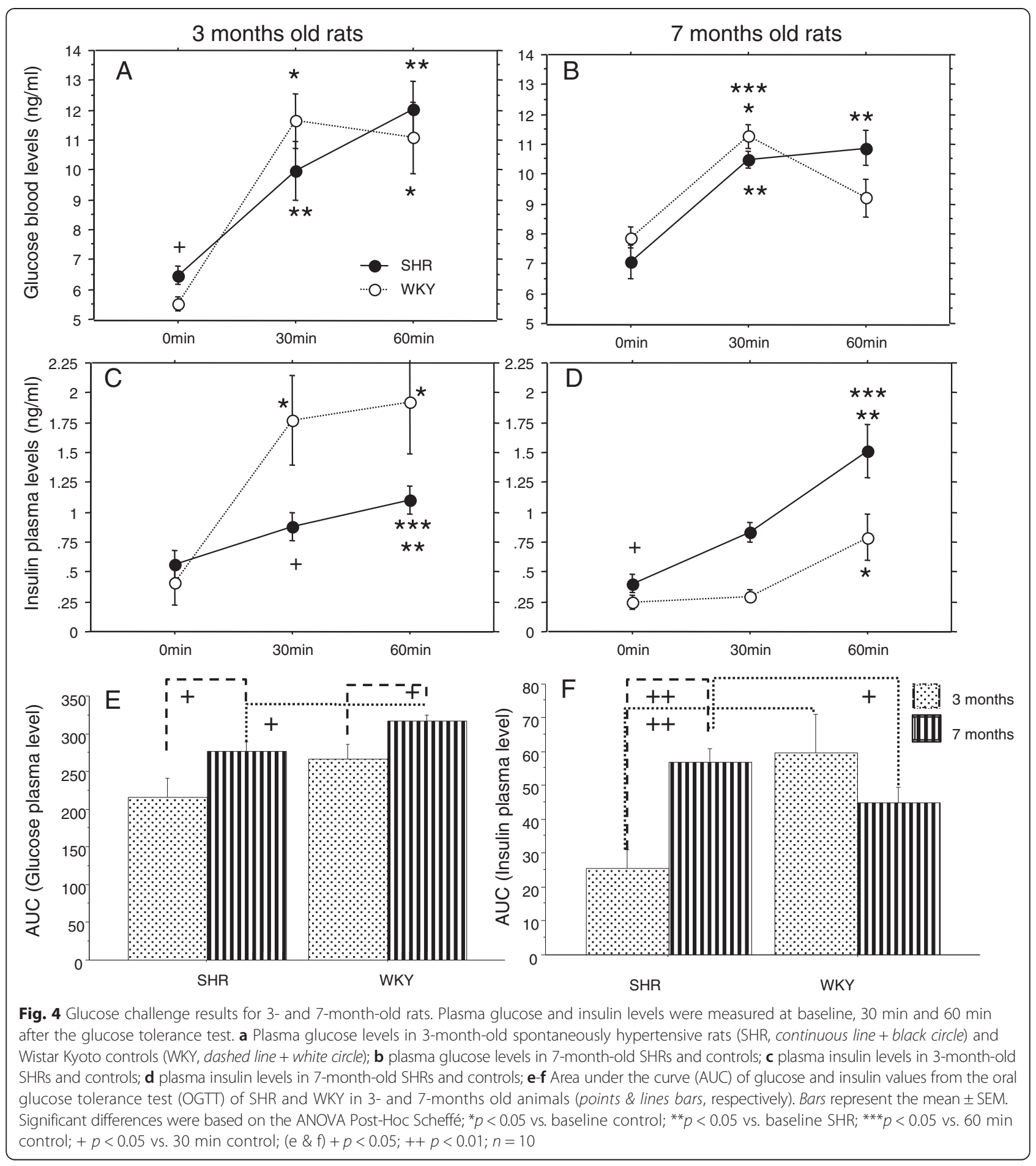

impairment of 1- and 3-month-old male SHRs compared to both these control strains.

A growing body of evidence points to the association of cognitive deficits and insulin resistance in humans and in animals [47-49], an issue that has been poorly explored in the SHR model; a presence of systemic insulin resistance in SHRs has been manifested by a plasma insulin response in 7 week-old animals (sex not specified) [2], and indirectly by a reduced insulin-stimulated glucose transporter bioavailability in adipocytes [50], while an unchanged expression of insulin receptor in the brain of 3-month-old male SHRs was reported by Yang et al. [51]. Our results indicate that the plasma insulin response to an oral glucose challenge is altered in SHRs 
compared to WKY but that the direction of change depends on a respective time-point of its measurement; lower plasma insulin response in 3-month $(\mathrm{AUC}=25$ / SHR vs. 60/WKY) and greater in 7-month-old female SHRs (AUC $=58 / \mathrm{SHR}$ vs. $45 / \mathrm{WKY}$ ) despite the similarity in the total serum glucose response in SHRs and WKYs. Such a difference in insulin AUC after OGTT was not detected in a recent study investigating various combinations of metabolic syndrome and $\mathrm{AD}$ markers in male SHR animals [51], indicating that a possible gender influence in this model might play a role not only in cognition but also in insulin response. Additionally, it could not be excluded that this time-dependent insulin response might correlate with an observed tendency for age-dependent worsening of learning and memory functions in female SHRs, indirectly also reflecting a difference in metabolic response between early (3-month-old) and chronic (7-month-old) hypertension.

Although several studies have suggested that learning and memory deficits observed in SHR animals were independent of hypertension [30, 40,52], others did find correlations between hypertension and cognitive deficits $[5,6,41,53]$. Having in mind that aging, female gender, and hypertension are considered risk factors for dementia, particularly of the Alzheimer's type (see metaanalysis in www.alzrisk.org and [54-57], usage of aged SHR animals with reduced cognitive performance might be worth considering as a possible model of a certain type of dementia [5, 58].

Several findings in SHR animals point to their susceptibility to metabolic alterations in the brain. For example, recent findings of Ritz et al. [59] showed that gene expression alterations in the cortex of 2-month-old (pre-hypertension) and 9-month-old SHRs (compared to WKY) pointed to a vulnerability to high-energy requirements in these animal models. Furthermore, studies of cerebral glucose utilization in SHR compared to WKY were found to be lower at aged animals (around 6 months) $[60,61]$ while not different in younger ones (12 weeks only) [62, 63]. Gene expression profiling in cell cultures originating from the brain stems of SHR and WKY animals revealed significantly enriched genes belonging to molecular pathways of the ATPase activity [64], further supporting the involvement of energy metabolism dysfunction in this model.

Glucose/energy hypometabolism in the brain might be a consequence of an insulin resistant brain state $[65,66]$, which seems to be involved in cognitive decline, as suggested in particular in AD and its animal models [9-11, 47, 67]. This hypothesis has been recently discussed for AD patients pointing to the pathophysiological importance of the vicious cycle of glucose/energy demand, vascular cognitive impairment, dementia, and aging, suggesting that an understanding of how vascular and metabolic factors interfere with a progressive loss of functional neuronal networks becomes essential for developing efficient drugs to prevent cognitive decline in the elderly [68]. Keeping in mind that glucose metabolism in the brain is under control of brain IR [12], our findings of dysfunction in brain IR and its downstream signaling cascade might contribute to the pathophysiological mechanisms of the cerebral glucose hypometabolism in SHRs reported in the literature [60]. We generally found lower total levels of the IR $\beta$ subunit in SHRs compared to WKYs in all brain regions investigated, regardless of a rat's age. This finding might indicate that SHRs have a general deficit in brain insulin signaling pathways. Furthermore, we observed an increased IR activity in the 7-month-old SHRs, measured as the ratio of phosphorylated to total IR $\beta$ subunits, which might represent a compensatory mechanism covering for reduced IR $\beta$ level. Yang and colleagues [51] could not find difference in IR, which, however, was measured in the whole brain (and not in specific brain regions as in our experiment) of young male SHR animals compared to WKY. Our findings demonstrated that GSK3 $\beta$ activity (indirectly measured by $\mathrm{p} /$ total GSK3 $\beta$ ratio), which is down-stream to IR signaling and is linked to hyperphosphorylation of tau-protein [69], increased with aging in the cerebral cortex of both the SHR and WKY group, while in the hippocampus it was significantly increased only in 7-month-old female SHRs compared to corresponding WKYs. In recent years, the over-activation of GSK3 $\beta$ in the brain has been reported to be involved in the pathophysiology of $\mathrm{AD}$ and of type-2 diabetes mellitus [70].

These results further support the hypothesis that the SHR model develops insulin resistance in the brain, manifested both at the level of IR protein/activity and its downstream signaling pathway, as well at the periphery. Moreover, our results obtained in the same pool of animals demonstrate for the first time the co-existence of hypertension with peripheral and central insulin resistance and cognitive deficits in SHR model.

\section{Conclusions}

This study provides the first combined evidence that cognitive deficits in the SHR model are accompanied by insulin-signaling dysfunction in the brain in parallel to the existence of peripheral insulin resistance. Factors like rat strain used for comparison, SHRs age and/or hypertension duration as well as sex should be considered in the interpretation of cognitive and metabolic performance of the SHR model. Further studies are required to address the question of a possible causal relationship between these metabolic and cognitive impairments in SHRs and accordingly explore whether SHRs might be also considered as a model of insulin resistance-induced type of dementia, in addition to being a model of hypertension and ADHD. 


\section{Availability of supporting data}

The three data sets supporting the results of this article are included within the article (and its additional files). 1) Additional file 1: Detailed cognitive testing methods; 2) Additional file 2: Figure S2: Comparison of the cognitive performance in the Morris Water Maze (MWM) training trials; 3) Additional file 3: Figure S3: The effect of sex and age on the cognitive performance of spontaneously hypertensive rats (SHR).

\section{Additional files}

\section{Additional file 1: Detailed cognitive testing methods. Additional file 2: Comparison of the cognitive performance in the Morris Water Maze (MWM) training trials. \\ Additional file 3: The effect of sex and age on the cognitive performance of spontaneously hypertensive rats (SHR).}

\section{Abbreviations}

AD: Alzheimer's disease; ADHD: Attention-deficit hyperactivity disorder; AUC: Area under the curve; CBF: Cerebral blood flow; Cl: Confidence interval; CNS: Central nervous system; ELISA: Enzyme-Linked Immunosorbent Assay; GSK3: Glycogen synthase-kinase-3; IR: Insulin receptor; IR $\beta$ : IR $\beta$-subunit; MWM: Morris Water Maze; MANOVA: Multifactorial analysis of variance; OGTT: Oral glucose tolerance test; PAT: Passive Avoidance Test; Akt/PKB: Protein kinase B; SHR: Spontaneously hypertensive rat; SD: Sprague-Dawley; STZ: Streptozotocin; WKY: Wistar-Kyoto rat strain.

\section{Competing interests}

The authors declare that they have no competing interests.

\begin{abstract}
Authors' contributions
JB, DII carried out the ELISA assays and performed the statistical analysis. AK carried out the behavior tests. VT performed the statistical analysis. PR \& SW participated in the design of the study, in the data interpretation and helped to draft the manuscript. EG and MSP conceived the study, participated in its design and coordination and drafted the manuscript. All authors read and approved the final manuscript.
\end{abstract}

\section{Authors' information}

Susanne Walitza and Melita Salkovic-Petrisic have equal senior authorship.

\section{Acknowledgment}

The authors would like to thank the "Bundesprogramm Chancengleichheit" and the national project, financed by the Croatian Ministry of Science and Education (MZOS), for their support. We also extend special thanks to Miss Miryame Hofmann and Mrs. Bozica Hrzan for technical assistance.

\begin{abstract}
Author details
${ }^{1}$ University Clinics of Child and Adolescent Psychiatry, University of Zurich, Neumuensterallee 9, 8032 Zurich, Switzerland. Neuroscience Center Zurich, University of Zurich and ETH Zurich, Zurich, Switzerland. ${ }^{3}$ University Hospital, Clinic and Policlinic for Psychiatry, Psychosomatic and Psychotherapy, University of Würzburg, Füchsleinstr. 15, D-97080 Würzburg, Germany. ${ }^{4}$ Department of Pharmacology and Croatian Institute for Brain Research, School of Medicine, University of Zagreb, Salata 11, 10000 Zagreb, Croatia. ${ }^{5}$ University Clinics of Child and Adolescent Psychiatry, University of Zurich, Wagistrasse 12, CH-8952 Schlieren, Switzerland.
\end{abstract}

Received: 20 April 2015 Accepted: 29 May 2015

Published online: 04 June 2015

\section{References}

1. Okamoto K, Aoki K, Nosaka S, Fukushima M. Cardiovascular diseases in the spontaneously hypertensive rat. Jpn Circ J. 1964;28:943-52.
2. Mondon CE, Reaven GM. Evidence of abnormalities of insulin metabolism in rats with spontaneous hypertension. Metabolism. 1988;37(4):303-5. doi:10.1016/0026-0495(88)90127-8.

3. Sagvolden T, Johansen EB, Woien G, Walaas SI, Storm-Mathisen J, Bergersen $\mathrm{LH}$, et al. The spontaneously hypertensive rat model of ADHD-the importance of selecting the appropriate reference strain. Neuropharmacology. 2009;57(7-8):619-26. doi:10.1016/j.neuropharm.2009.08.004.

4. Sontag TA, Tucha O, Walitza S, Lange KW. Animal models of attention deficit/hyperactivity disorder (ADHD): a critical review. Atten Defic Hyperact Disord. 2010;2(1):1-20. doi:10.1007/s12402-010-0019-x.

5. Meneses A, Perez-Garcia G, Ponce-Lopez T, Tellez R, Gallegos-Cari A, Castillo C. Spontaneously hypertensive rat (SHR) as an animal model for ADHD: a short overview. Rev Neurosci. 2011;22(3):365-71. doi:10.1515/RNS.2011.024.

6. Sabbatini M, Catalani A, Consoli C, Marletta N, Tomassoni D, Avola R. The hippocampus in spontaneously hypertensive rats: an animal model of vascular dementia? Mech Ageing Dev. 2002;123(5):547-59.

7. Terry Jr AV, Hernandez CM, Buccafusco JJ, Gattu M. Deficits in spatial learning and nicotinic-acetylcholine receptors in older, spontaneously hypertensive rats. Neuroscience. 2000;101(2):357-68.

8. Meneses A, Castillo C, Ibarra M, Hong E. Effects of aging and hypertension on learning, memory, and activity in rats. Physiol Behav. 1996;60(2):341-5.

9. Salkovic-Petrisic M, Osmanovic J, Grünblatt E, Riederer P, Hoyer S. Modeling sporadic Alzheimer's disease: the insulin resistant brain state generates multiple long-term morphobiological abnormalities including hyperphosphorylated tau protein and amyloid-beta. J Alzheimers Dis. 2009;18(4):729-50. doi:10.3233/JAD-2009-1184.

10. Lester-Coll N, Rivera EJ, Soscia SJ, Doiron K, Wands JR, de la Monte SM. Intracerebral streptozotocin model of type 3 diabetes: relevance to sporadic Alzheimer's disease. J Alzheimers Dis. 2006;9(1):13-33.

11. de la Monte SM. Brain insulin resistance and deficiency as therapeutic targets in Alzheimer's disease. Curr Alzheimer Res. 2012;9(1):35-66.

12. Heni M, Hennige AM, Peter A, Siegel-Axel D, Ordelheide AM, Krebs N, et al. Insulin promotes glycogen storage and cell proliferation in primary human astrocytes. PLoS One. 2011;6(6):e21594. doi:10.1371/journal.pone.0021594. PONE-D-10-03525.

13. Liu Y, Liu F, Grundke-lqbal I, lqbal K, Gong CX. Deficient brain insulin signalling pathway in Alzheimer's disease and diabetes. J Pathol. 2011;225(1):54-62. doi:10.1002/path.2912.

14. Deng Y, Li B, Liu Y, lqbal K, Grundke-lqbal I, Gong CX. Dysregulation of insulin signaling, glucose transporters, O-GlcNAcylation, and phosphorylation of tau and neurofilaments in the brain: Implication for Alzheimer's disease. Am J Pathol. 2009;175(5):2089-98. doi:10.2353/ajpath.2009.090157.

15. Bartl J, Monoranu CM, Wagner AK, Kolter J, Riederer P, Grunblatt E. Alzheimer's disease and type 2 diabetes: two diseases, one common link? World J Biol Psychiatry. 2013;14(3):233-40. doi:10.3109/15622975.2011.650204.

16. Qu Z, Jiao Z, Sun $X$, Zhao Y, Ren J, Xu G. Effects of streptozotocin-induced diabetes on tau phosphorylation in the rat brain. Brain Res. 2011;1383:300-6. doi:10.1016/j.brainres.2011.01.084.

17. Chen Y, Cao CP, Li CR, Wang W, Zhang D, Han LL, et al. Ghrelin modulates insulin sensitivity and tau phosphorylation in high glucose-induced hippocampal neurons. Biol Pharm Bull. 2010;33(7):1165-9.

18. Lopez-Gil X, Amat-Roldan I, Tudela R, Castane A, Prats-Galino A, Planas $A M$, et al. DWI and complex brain network analysis predicts vascular cognitive impairment in spontaneous hypertensive rats undergoing executive function tests. Front Aging Neurosci. 2014;6:167. doi:10.3389/fnagi.2014.00167.

19. Shaul ME, Hallacoglu B, Sassaroli A, Shukitt-Hale B, Fantini S, Rosenberg $\mathbb{H}_{\text {, }}$ et al. Cerebral blood volume and vasodilation are independently diminished by aging and hypertension: a near infrared spectroscopy study. J Alzheimers Dis. 2014;42 Suppl 3:S189-98. doi:10.3233/JAD-132504.

20. Clements KM, Saunders AJ, Robertson BA, Wainwright PE. Spontaneously hypertensive, Wistar Kyoto and Sprague-Dawley rats differ in their use of place and response strategies in the water radial arm maze. Neurobiol Learn Mem. 2007:87(2):285-94. doi:10.1016/j.nlm.2006.09.003.

21. Ferguson SA, Cada AM. Spatial learning/memory and social and nonsocial behaviors in the spontaneously hypertensive, Wistar-Kyoto and SpragueDawley rat strains. Pharmacol Biochem Behav. 2004;77(3):583-94. doi:10.1016/j.pbb.2003.12.014.

22. Langen B, Dost R. Comparison of SHR, WKY and Wistar rats in different behavioural animal models: effect of dopamine D1 and alpha2 agonists. Atten Defic Hyperact Disord. 2011;3(1):1-12. doi:10.1007/s12402-010-0034-y. 
23. Robertson BA, Clements KM, Wainwright PE. The working memory capabilities of the spontaneously hypertensive rat. Physiol Behav. 2008;94(3):481-6. doi:10.1016/j.physbeh.2008.02.016.

24. Sontag TA, Fuermaier AB, Hauser J, Kaunzinger I, Tucha O, Lange KW. Spatial memory in spontaneously hypertensive rats (SHR). PLoS One. 2013;8(8):e74660. doi:10.1371/journal.pone.0074660.

25. Wyss JM, Chambless BD, Kadish I, van Groen T. Age-related decline in water maze learning and memory in rats: strain differences. Neurobiol Aging. 2000;21(5):671-81.

26. van den Bergh FS, Bloemarts E, Chan JS, Groenink L, Olivier B, Oosting RS. Spontaneously hypertensive rats do not predict symptoms of attentiondeficit hyperactivity disorder. Pharmacol Biochem Behav. 2006;83(3):380-90. doi:10.1016/j.pbb.2006.02.018.

27. Chelaru MI, Yang PB, Dafny N. Sex differences in the behavioral response to methylphenidate in three adolescent rat strains (WKY, SHR, SD). Behav Brain Res. 2012;226(1):8-17. doi:10.1016/j.bbr.2011.08.027.

28. Hendley ED, Wessel DJ, Atwater DG, Gellis J, Whitehorn D, Low WC. Age, sex and strain differences in activity and habituation in SHR and WKY rats. Physiol Behav. 1985;34(3):379-83.

29. Pires VA, Pamplona FA, Pandolfo P, Fernandes D, Prediger RD, Takahashi RN. Adenosine receptor antagonists improve short-term object-recognition ability of spontaneously hypertensive rats: a rodent model of attention-deficit hyperactivity disorder. Behav Pharmacol. 2009;20(2):134-45. doi:10.1097/FBP.0b013e32832a80bf.

30. Prediger RD, Pamplona FA, Fernandes D, Takahashi RN. Caffeine improves spatial learning deficits in an animal model of attention deficit hyperactivity disorder (ADHD) - the spontaneously hypertensive rat (SHR). Int J Neuropsychopharmacol. 2005;8(4):583-94. doi:10.1017/S1461145705005341.

31. Robinson AM, Hopkins ME, Bucci DJ. Effects of physical exercise on ADHD-like behavior in male and female adolescent spontaneously hypertensive rats. Dev Psychobiol. 2011;53(4):383-90. doi:10.1002/dev.20530.

32. Bucci DJ, Hopkins ME, Keene CS, Sharma M, Orr LE. Sex differences in learning and inhibition in spontaneously hypertensive rats. Behav Brain Res. 2008;187(1):27-32. doi:10.1016/j.bbr.2007.08.022.

33. Andersen SL. Changes in the second messenger cyclic AMP during development may underlie motoric symptoms in attention deficit/ hyperactivity disorder (ADHD). Behav Brain Res. 2002;130(1-2):197-201.

34. Hinojos CA, Boerwinkle E, Fornage M, Doris PA. Combined genealogical, mapping, and expression approaches to identify spontaneously hypertensive rat hypertension candidate genes. Hypertension. 2005;45(4):698-704. doi:10.1161/01.HYP.0000156498.78896.37.

35. Luo Y, Owens D, Mulder G, McVey A, Fisher T. Blood Pressure Characterization of Hypertensive and Control Rats for Cardiovascular Studies. AHA, Atlanta: Charles River; 2008.

36. Salkovic-Petrisic M, Osmanovic-Barilar J, Knezovic A, Hoyer S, Mosetter K, Reutter W. Long-term oral galactose treatment prevents cognitive deficits in male Wistar rats treated intracerebroventricularly with streptozotocin. Neuropharmacology. 2014;77:68-80. doi:10.1016/j.neuropharm.2013.09.002.

37. Salkovic-Petrisic M, Tribl F, Schmidt M, Hoyer S, Riederer P. Alzheimer-like changes in protein kinase $B$ and glycogen synthase kinase-3 in rat frontal cortex and hippocampus after damage to the insulin signalling pathway. J Neurochem. 2006;96(4):1005-15.

38. Paxinos $\mathrm{G}$, Watson $\mathrm{C}$. The rat brain in stereotaxic coordinates. Compact sixth edition ed. London, UK: Elsevier Inc.; 2009.

39. Bradford MM. Rapid and sensitive method for quantitation of microgram quantities of proteins utilizing principle of protein dye binding. Anal Biochem. 1976;72:248-54.

40. Kantak KM, Singh T, Kerstetter KA, Dembro KA, Mutebi MM, Harvey RC, et al. Advancing the spontaneous hypertensive rat model of attention deficit/hyperactivity disorder. Behav Neurosci. 2008;122(2):340-57. doi:10.1037/0735-7044.122.2.340.

41. Hernandez CM, Hoifodt H, Terry Jr AV. Spontaneously hypertensive rats: further evaluation of age-related memory performance and cholinergic marker expression. J Psychiatry Neurosci. 2003;28(3):197-209.

42. Terry Jr AV, Buccafusco JJ. The cholinergic hypothesis of age and Alzheimer's disease-related cognitive deficits: recent challenges and their implications for novel drug development. J Pharmacol Exp Ther. 2003;306(3):821-7.

43. Alsop B. Reprint of "Problems with spontaneously hypertensive rats (SHR) as a model of attention-deficit/hyperactivity disorder (AD/HD)". J Neurosci Methods. 2007;166(2):XV-XXI. doi:10.1016/j.jneumeth.2006.12.019.
44. Bucci DJ, Hopkins ME, Nunez AA, Breedlove SM, Sisk CL, Nigg JT. Effects of sex hormones on associative learning in spontaneously hypertensive rats. Physiol Behav. 2008;93(3):651-7. doi:10.1016/j.physbeh.2007.11.005.

45. Gattu M, Pauly JR, Boss KL, Summers JB, Buccafusco JJ. Cognitive impairment in spontaneously hypertensive rats: role of central nicotinic receptors. I Brain Res. 1997;771(1):89-103.

46. Gattu M, Terry Jr AV, Pauly JR, Buccafusco JJ. Cognitive impairment in spontaneously hypertensive rats: role of central nicotinic receptors. Part II. Brain Res. 1997;771(1):104-14.

47. De Felice FG, Lourenco MV, Ferreira ST. How does brain insulin resistance develop in Alzheimer's disease? Alzheimers Dement. 2014;10(1S):S26-32. doi:10.1016/j.jalz.2013.12.004.

48. Dineley KT, Jahrling JB, Denner L. Insulin resistance in Alzheimer's disease. Neurobiol Dis. 2014;72(Pt A):92-103. doi:10.1016/j.nbd.2014.09.001.

49. Crichton GE, Elias MF, Buckley JD, Murphy KJ, Bryan J, Frisardi V. Metabolic syndrome, cognitive performance, and dementia. J Alzheimers Dis. 2012;30 Suppl 2:S77-87. doi:10.3233/JAD-2011-111022.

50. Collison M, James DJ, Graham D, Holman GD, Connell JM, Dominiczak AF, et al. Reduced insulin-stimulated GLUT4 bioavailability in stroke-prone spontaneously hypertensive rats. Diabetologia. 2005;48(3):539-46. doi:10.1007/s00125-005-1674-x.

51. Yang HT, Sheen YJ, Kao CD, Chang CA, Hu YC, Lin JL. Association between the characteristics of metabolic syndrome and Alzheimer's disease. Metab Brain Dis. 2013;28(4):597-604. doi:10.1007/s11011-013-9406-2.

52. Loskutova LV, Dubrovina NI, Markel AL. Comparative analysis of the persistence of a conditioned passive avoidance reflex in rats with different forms of inherited hypertension. Neurosci Behav Physiol. 2007;37(6):577-82 doi:10.1007/s11055-007-0055-y.

53. Chan SH, Wu KL, Kung PS, Chan JY. Oral intake of rosiglitazone promotes a central antihypertensive effect via upregulation of peroxisome proliferatoractivated receptor-gamma and alleviation of oxidative stress in rostral ventrolateral medulla of spontaneously hypertensive rats. Hypertension. 2010;55(6):1444-53. doi:10.1161/HYPERTENSIONAHA.109.149146.

54. Power MC, Weuve J, Gagne JJ, McQueen MB, Viswanathan A, Blacker D. The association between blood pressure and incident Alzheimer disease: a systematic review and meta-analysis. Epidemiology. 2011;22(5):646-59. doi:10.1097/EDE.0b013e31822708b5.

55. World Health Organization. Dementia. World Health Organization. 2012. http://www.who.int/mediacentre/factsheets/fs362/en/index.html. Accessed 17.09.2012 2012.

56. Azad NA, Al Bugami M, Loy-English I. Gender differences in dementia risk factors. Gend Med. 2007:4(2):120-9.

57. Mukhtar O, Jackson SH. The hypertension in the very elderly trial - latest data. Br J Clin Pharmacol. 2013;75(4):951-4. doi:10.1111/j.1365-2125.2012.04427.x.

58. Tayebati SK, Tomassoni D, Amenta F. Spontaneously hypertensive rat as a model of vascular brain disorder: microanatomy, neurochemistry and behavior. J Neurol Sci. 2012;322(1-2):241-9. doi:10.1016/j.jns.2012.05.047.

59. Ritz MF, Grond-Ginsbach C, Engelter S, Lyrer P. Gene expression suggests spontaneously hypertensive rats may have altered metabolism and reduced hypoxic tolerance. Curr Neurovasc Res. 2012;9(1):10-9.

60. Wei L, Lin SZ, Tajima A, Nakata H, Acuff V, Patlak C, et al. Cerebral glucose utilization and blood flow in adult spontaneously hypertensive rats. Hypertension. 1992;20(4):501-10.

61. Johansson BB. Pentoxifylline: cerebral blood flow and glucose utilization in conscious spontaneously hypertensive rats. Stroke. 1986;17(4):744-7.

62. Orzi F, Morisco C, Colangelo V, Di Grezia R, Lembo G. Lack of effect of insulin on glucose utilization of the hypothalamus in normotensive and hypertensive rats. Neurosci Lett. 2000;278(1-2):29-32.

63. Kadekaro M, Savaki HE, Kutyna FA, Davidsen L, Sokoloff L. Metabolic mapping in the sympathetic ganglia and brain of the spontaneously hypertensive rat. J Cereb Blood Flow Metab. 1983;3(4):460-7. doi:10.1038/jcbfm.1983.72.

64. Ferrari MF, Reis EM, Matsumoto JP, Fior-Chadi DR. Gene expression profiling of cultured cells from brainstem of newborn spontaneously hypertensive and Wistar Kyoto rats. Cell Mol Neurobiol. 2009;29(3):287-308. doi:10.1007/s10571-008-9321-y.

65. Sievenpiper JL, Jenkins AL, Whitham DL, Vuksan V. Insulin resistance: concepts, controversies, and the role of nutrition. Can J Diet Pract Res. 2002;63(1):20-32.

66. Hoyer S. Glucose metabolism and insulin receptor signal transduction in Alzheimer disease. Eur J Pharmacol. 2004;490(1-3):115-25. 
67. Craft S, Cholerton B, Baker LD. Insulin and Alzheimer's disease: untangling the web. J Alzheimers Dis. 2013;33 Suppl 1:S263-75. doi:10.3233/JAD-2012-129042.

68. Popa-Wagner A, Buga AM, Popescu B, Muresanu D. Vascular cognitive impairment, dementia, aging and energy demand. A vicious cycle. J Neural Transm. 2013;In Print. doi:10.1007/s00702-013-1129-3.

69. Hernandez F, Lucas JJ, Avila J. GSK3 and tau: two convergence points in Alzheimer's disease. J Alzheimers Dis. 2013;33 Suppl 1:S141-4. doi:10.3233/JAD-2012-129025.

70. Gao C, Holscher C, Liu Y, Li L. GSK3: a key target for the development of novel treatments for type 2 diabetes mellitus and Alzheimer disease. Rev Neurosci. 2011;23(1):1-11. doi:10.1515/rns.2011.061.

\section{Submit your next manuscript to BioMed Central and take full advantage of:}

- Convenient online submission

- Thorough peer review

- No space constraints or color figure charges

- Immediate publication on acceptance

- Inclusion in PubMed, CAS, Scopus and Google Scholar

- Research which is freely available for redistribution 